Ensino, S aúde e Ambiente-V4 (2), pp. 17-27, ago. 2011

\title{
HISTÓRIA DA MATEMÁTICA E MESTRADO PROFISSIONAL: UMA FUSÃO POSSÍVEL
}

\section{HISTORY OF MATHEMATICS AND PROFESSIONAL MASTERS DEGREE: A POSSIBLE MERGER}

\author{
Bernadete Barbosa Morey ${ }^{1}$ e Severino Carlos Gomes ${ }^{2}$ \\ ${ }^{1}$ UFRN, bernadetemorey@ gmail.com \\ ${ }^{2}$ IFRN, severino.gomes@ifrn.edu.br
}

\section{RESUMO}

Nos últimos anos, uma nova modalidade de pós-graduação surgiu nas instituições de ensino superior: o mestrado profissional. A História da Matemática, por sua vez, como ferramenta no ensino de Matemática não é uma ideia nova e estudos relacionados a esta temática tem recebido a adesão de pesquisadores em todo o mundo nas últimas décadas. No entanto, os estudos pós-graduados relacionados à História da Matemática em sua imensa maioria têm se dado no mestrado e doutorado em sua modalidade acadêmica. É nova a ideia, pelo menos nas universidades brasileiras, de trazer a História da Matemática para o mestrado profissional. Tendo em vista estas considerações, esse artigo relata estudo enfocando a fusão possível entre a abordagem histórica no ensino de Matemática e o mestrado profissional. Para isso são feitas considerações sobre a História da Matemática enquanto metodologia de ensino, uma caracterização sobre o que venha a ser um mestrado profissional e sua diferença para o mestrado acadêmico. Por fim, o texto apresenta um exemplo do que venha a ser essa fusão entre História da Matemática, conteúdo matemático escolar e mestrado profissional.

Palavras-chave: História da Matemática, Mestrado Profissional, Ensino, Trigonometria.

\begin{abstract}
In recent years a new type of research emerged in institutions of higher education: the professional master's degree. The History of Mathematics, in turn, as a tool in teaching Mathematics is not a new idea and studies related to this subject has received the adherence of researchers around the world in recent decades. However, post-graduate studies related to the History of Mathematics in their vast majority have been in the master's and doctoral degrees in their academic mode. It's a new idea, at least in brazilian universities to bring the History of Mathematics for the professional master. With these considerations, this article aims to present the research, focusing on possible links between the historical approach to the teaching of mathematics and master's courses. For this we discuss the History of Mathematics as a teaching methodology and characterization of this modality masters. Finally, the text presents an example that will be a fusion between History of Mathematics and mathematical content of school as an end product of a professional master.
\end{abstract}

Keywords: History of Mathematics, The Professional Master's Degree, Teaching, Trigonometry. 


\section{INTRODUÇÃO}

Nas últimas décadas, diversos encontros, seminários e congressos com educadores matemáticos, têm sido realizados com o intuito de se discutir o processo de ensino e de aprendizagem da Matemática. Nesses momentos, são feitas reflexões, debates e propostas visando à melhoria no ensino de tal ciência. Uma das alternativas consideradas atualmente é o ensino de Matemática através da abordagem histórica. São muitos os pesquisadores em Educação Matemática que apóiam a História da Matemática como importante na formação do estudante, em qualquer nível de ensino.

Sobre essa importância, Ferreira (2001, p. 15) argumenta que a História da Matemática “[...] dá ao aluno a noção exata desta ciência, como uma ciência em construção, com erros e acertos e sem verdades universais." Ou seja, através da História o estudante passa a conhecer a Matemática como um saber que tem significado dentro de um contexto e que foi, e está sendo, construído pela necessidade de cada época.

Porém, mesmo admitindo que a História da Matemática traga benefícios para o ensino, duas questões são motivo de discussões entre os educadores matemáticos. A primeira delas diz respeito a como está sendo a preparação dos professores de Matemática para a utilização dessa abordagem; e a segunda se refere às estratégias de ensino pertinentes e como devem ser aplicadas. Ambas as questões estão dentro de nosso foco de interesse por ser esta a escolha que fizemos em nossos estudos no mestrado profissional.

No presente artigo vamos discutir sobre a distinção entre o mestrado acadêmico e o profissional, sobre a História da Matemática como uma ferramenta de ensino e a seguir mostraremos o caminho que escolhemos para fazer a fusão dos estudos em História da Matemática e o mestrado profissional.

\section{A HISTÓRIA DA MATEMÁTICA NO ENSINO DA MATEMÁTICA}

Nos últimos anos tem havido um interesse crescente no papel da História da Matemática no ensino e na aprendizagem da Matemática. Diversos estudiosos, em vários países, pesquisam sobre o papel da História da Matemática, em suas várias dimensões, em todos os níveis do sistema educacional; em suas relações com o ensino e a aprendizagem da Matemática; bem como no que diz respeito à formação do professor. 
A divulgação dessas pesquisas vem impulsionando os estudos referentes às potencialidades do uso da História no ensino. A justificativa para esse crescente interesse reside no fato de que

Em diversas situações, tendo a matemática como um recurso, pode se esclarecer ideias matemáticas que estão sendo construídas por estudantes, especialmente para dar respostas a algumas perguntas e, desta forma, contribuir para a constituição de um olhar crítico sobre os objetos do conhecimento. (FASANELLI, 2002, p. 4, tradução nossa).

Ou seja, a História da Matemática pode transformar (ou não) uma situação de ensino tradicional, independente do nível de ensino, em um processo exploratório, contextualizado no qual professor e estudante participam ativamente analisando e discutindo os processos de formação do conhecimento no passado atrelados ao desenvolvimento intelectual no presente.

A forma como a História da Matemática pode ser usada em sala de aula deve variar de acordo com o nível de ensino, pois os estudantes, nas diversas faixas etárias, têm necessidades e possibilidades diferentes. Já no ensino básico, a investigação da evolução matemática, dentro de contextos sócio-culturais, pode conduzir à constatação de que todas as culturas humanas têm dado origem a desenvolvimentos matemáticos que estão agora à disposição de todos. No ensino superior, a História pode contribuir no desenvolvimento de hábitos de leitura, às vezes negligenciados nos cursos de formação ou aperfeiçoamento de professores.

Considerando estes aspectos, a História da Matemática pode ser usada como fonte de ensino através do estudo da biografia dos matemáticos, da origem e significado dos termos matemáticos, dos textos originais, dos problemas históricos e da reconstrução histórica dos conceitos matemáticos. No entanto, a maioria dos professores das escolas necessitam de especial apoio para introduzir em sua sala de aula uma nova abordagem. Este é o caso da introdução da História da Matemática no processo de ensino, como podemos ler no artigo de autoria de Siu (2004/2007).

Neste artigo Siu mostra que, de acordo com dados obtidos num estudo com 360 professores de 41 escolas, os professores consideram importante o uso da História da Matemática na sala de aula, porém, estes mesmo professores declaram que eles não lançam mão deste recurso. Siu, neste mesmo artigo, nos fornece dados obtidos de 608 
respondentes, todos eles professores de Matemática em formação ou em formação continuada que apontam pelo menos 15 razões para eles não usarem a História da Matemática em suas aulas. Algumas destas razões apontadas gozam de mais unanimidade e, já outras, de menos. Aqui iremos focalizar apenas duas delas:

1. Falta de material adequado (citado por $64.47 \%$ dos respondentes).

2. Falta de formação adequada (citado por $82.89 \%$ dos respondentes).

Apoiados por resultados apresentados em publicações como a mencionada acima e em nossa experiência de anos trabalhando com professores em formação foi que decidimos buscar meios de amenizar as lacunas a que se refere os pontos 1 e 2 citados.

\title{
MESTRADO PROFISSIONAL E HISTÓRIA DA MATEMÁTICA
}

Vejamos agora o que dizem os documentos legais que regem a criação do mestrado profissional no âmbito das instituições de ensino superior:

\begin{abstract}
"Mestrado Profissional" é a designação do Mestrado que enfatiza estudos e técnicas diretamente voltadas ao desempenho de um alto nível de qualificação profissional. Esta ênfase é a única diferença em relação ao acadêmico. Confere, pois, idênticos grau e prerrogativas, inclusive para o exercício da docência, e, como todo programa de pósgraduação stricto sensu, tem a validade nacional do diploma condicionada ao reconhecimento prévio do curso (Parecer CNE/CES 0079/2002).
\end{abstract}

Ou seja, o mestrado profissional tem seu foco na realização de pesquisas para o desenvolvimento e aperfeiçoamento profissional. É uma capacitação diferenciada da oferecida no mestrado acadêmico. Enquanto o mestrado acadêmico se concentra no campo das pesquisas teóricas, o mestrado profissional prioriza as ações direcionadas para intervenções nas práticas de sala de aula.

Quanto ao trabalho de conclusão do mestrado profissional a exigência é que ele seja um estudo voltado par a prática de sala de aula e que, além disso, apresente de forma explícita e destacável um produto educacional. Este produto educacional pode ter vários formatos e vários objetivos, sendo que a exigência única é que se trate de alguma ferramenta que venha a ajudar o professor em sua prática profissional.

Aliando estas duas vertentes, ou seja, a busca de meios que auxiliem os professores de Matemática a introduzir a abordagem histórica em suas aulas de Matemática e, por outro lado, atender às exigências, aos princípios e aos objetivos do mestrado profissional, chegamos a um formato de produto educacional que consiste em 
uma sequência de atividades matemáticas nas quais a História da Matemática participa de formas e níveis distintos (ou seja, mais ou menos explicitamente), cujo objetivo é servir como material auxiliar ao professor em suas aulas de Matemática.

Tendo como ponto de partida os estudos de Brito e Morey (2004) sobre as dificuldades dos professores de Matemática em geometria e trigonometria, passamos a desenvolver uma sequência de ensino de trigonometria através da abordagem histórica, no qual os conteúdos trigonométricos e seu desenvolvimento histórico aparecem indissolúveis. A sequencia completa, composta de cinco atividades, começa com atividades de caráter mais geométrico e segue, paulatinamente, com atividades que exigem a introdução de novos conceitos, realização de cálculos, discussão em grupo de alunos, até chegar aos conceitos trigonométricos básicos, ou seja, o conceito de comprimento de corda e o de seu equivalente moderno, o conceito de seno.

Portanto, o conteúdo trigonométrico apresentado em nosso estudo prioriza o enfoque geométrico. Com isso, além de seguir o percurso histórico, tratamos a geometria como fundamental para estudos trigonométricos. Os procedimentos algébricos enfadonhos explorados em diversos livros didáticos de trigonometria não são abordados. Em seu lugar apresentamos questões em que a investigação e o raciocínio são ferramentas.

Levando em consideração os aspectos aqui citados, apresentamos na figura 1 uma síntese das atividades com seus objetivos e assuntos abordados.

\begin{tabular}{|c|c|c|c|}
\hline Atividade & Título & Objetivos & Assuntos abordados \\
\hline $\mathbf{1}^{\mathrm{a}}$ & $\begin{array}{l}\text { Explorando } \\
\text { polígonos } \\
\text { regulares } \\
\text { inscritos na } \\
\text { circunferência }\end{array}$ & $\begin{array}{l}\text { Relembrar conceitos, elementos e } \\
\text { propriedades dos polígonos } \\
\text { regulares inscritos em uma } \\
\text { circunferência. }\end{array}$ & $\begin{array}{l}\text { Circunferência e } \\
\text { polígonos regulares. }\end{array}$ \\
\hline $2^{\mathrm{a}}$ & $\begin{array}{l}\text { Calculando os } \\
\text { comprimentos de } \\
\text { algumas cordas }\end{array}$ & $\begin{array}{l}\text { Investigar relação entre as } \\
\text { medidas do ângulo central e do } \\
\text { lado de polígonos inscritos em } \\
\text { uma circunferência. Ainda, } \\
\text { determinar o comprimento de } \\
\text { algumas cordas. }\end{array}$ & $\begin{array}{l}\text { Circunferência, } \\
\text { polígonos regulares, } \\
\text { teorema de Pitágoras, } \\
\text { triângulos isósceles e } \\
\text { equilátero. }\end{array}$ \\
\hline $3^{\mathbf{a}}$ & $\begin{array}{l}\text { A transformação } \\
\text { da corda em seno }\end{array}$ & $\begin{array}{l}\text { Calcular o seno de um ângulo } \\
\text { através do valor da meia-corda. }\end{array}$ & $\begin{array}{c}\text { Triângulos, mediatriz de } \\
\text { um segmento e seno. }\end{array}$ \\
\hline $4^{a}$ & $\begin{array}{l}\text { O radiano como } \\
\text { unidade de } \\
\text { medida angular }\end{array}$ & $\begin{array}{l}\text { Conceituar o radiano como } \\
\text { unidade de medida e compará-lo } \\
\text { com o grau. }\end{array}$ & $\begin{array}{l}\text { Circunferência, } \\
\text { triângulos isósceles e } \\
\text { equilátero e unidades de } \\
\text { medidas de arcos. }\end{array}$ \\
\hline $5^{a}$ & $\begin{array}{l}\text { O seno na } \\
\text { circunferência } \\
\text { unitária }\end{array}$ & $\begin{array}{c}\text { Conceituar o seno na } \\
\text { circunferência trigonométrica e } \\
\text { estabelecer propriedades do seno }\end{array}$ & $\begin{array}{l}\text { Circunferência, } \\
\text { projeções ortogonais e } \\
\text { seno. }\end{array}$ \\
\hline
\end{tabular}


através do seu gráfico.

Figura 1 - Quadro resumo das atividades.

Na elaboração da sequência, tivemos sempre em mente o caminho percorrido pelo conhecimento trigonométrico desde a Antiguidade. Ou seja, nossas atividades têm base na geometria euclidiana necessária à transição para a trigonometria moderna.

Como exemplificação do trabalho desenvolvido, apresentamos na íntegra uma das atividades do caderno para o ensino de trigonometria numa abordagem histórica.

\section{Atividade 3: A transformação da corda em seno.}

$\mathrm{Na}$ atividade anterior você calculou o comprimento das cordas de $90^{\circ}, 60^{\circ}, 120^{\circ}$ e $72^{\circ}$. Estas cordas são lados de polígonos regulares inscritos numa circunferência. Também construiu uma pequena tabela de cordas baseada no Almagesto de Ptolomeu.

Durante vários séculos, os cálculos nas observações astronômicas dependiam da tabela de cordas de Ptolomeu. Com o texto denominado Surya Siddhanta (Sistema do Sol), os astrônomos hindus abriram novas perspectivas para a trigonometria por não seguir o mesmo caminho de Ptolomeu. Enquanto na astronomia grega se usava tábua de cordas, no Surya a correspondência se dava entre a metade da corda e a metade do ângulo central correspondente. Na figura 16 está representada a meia corda hindu chamada jya. (MAOR, 1998).

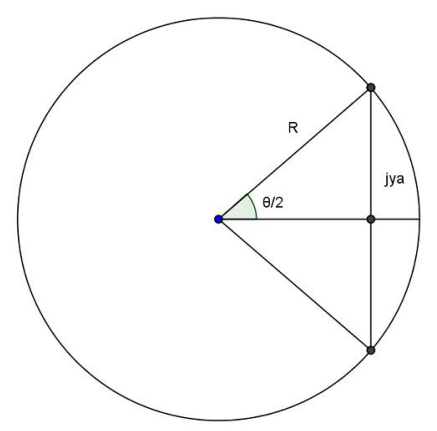

Figura 16: Meia corda hindu.

A palavra jya é uma das várias grafias para a palavra "corda" em hindu. Posteriormente os árabes a transliteraram para $j y b$, que depois foi incorretamente lida como jayb (seio), pelo tradutor Gerardo de Cremona (c. 1150). Traduzindo do árabe 
para o latim, ele usou o equivalente latino sinus, o que hoje usamos como seno. (KENNEDY, 1992).

Nesse momento, vamos nos deter a relação entre seno e comprimento da corda de um mesmo ângulo. Observe figura 17.

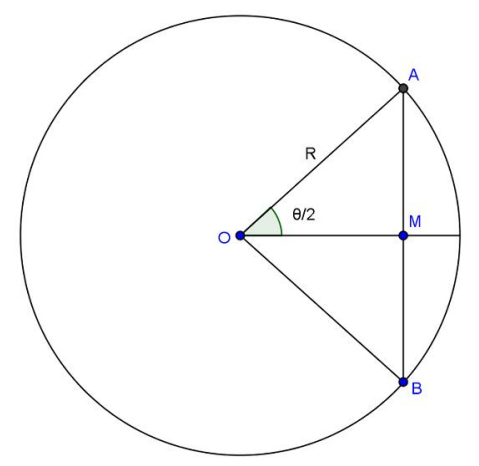

Legenda

$\theta / 2$ - metade do ângulo central AÔB

$\mathrm{R}$ - raio da circunferência

$\overline{\mathrm{AB}}$ - corda

AM - meia corda.

Figura 17: Relação entre corda e seno.

Nela, tem-se que:

$$
\operatorname{sen}\left(\frac{\theta}{2}\right)=\frac{A M}{O A}=\frac{2 A M}{2 O A}=\frac{A B}{20 A}=\frac{\operatorname{crd} \theta}{2 R}
$$

Com essa relação é possível construir uma tabela trigonométrica de senos partindo de uma tabela de cordas geométricas.

Você compreendeu todo processo de transformação do cálculo do comprimento das cordas até se chegar ao valor do seno?

Veja o seguinte exemplo para a determinação do seno de $30^{\circ}$. Nesse caso, o ângulo central $\theta$ tem abertura de $60^{\circ}$ e, de acordo com a tabela 2 , a corda de $60^{\circ}$ tem comprimento R. Então,

$$
\operatorname{sen} 30^{\circ}=\operatorname{sen}\left(\frac{60^{\circ}}{2}\right)=\frac{\operatorname{crd} 60^{\circ}}{2 \mathrm{R}}=\frac{\mathrm{R}}{2 \mathrm{R}}=\frac{1}{2}
$$

Ou seja, o seno de $30^{\circ}$ é $\frac{1}{2}$. Para auxiliar nas suas reflexões, tente resolver o desafio seguinte. 


\section{D eafio 4}

Com os valores obtidos para os comprimentos das cordas do ângulos $\boldsymbol{\theta}$, com a relação entre corda e seno apresentada anteriormente e com uma calculadora científica complete a tabela 4 .

Tabela 4: Relação entre corda e seno.

\begin{tabular}{|c|c|c|c|}
\hline $\boldsymbol{\theta}$ & $\operatorname{crd} \boldsymbol{\theta}$ & $\operatorname{sen}\left(\frac{\theta}{2}\right)$ & $\begin{array}{c}\operatorname{sen}\left(\frac{\theta}{2}\right) \\
\text { (calculadora) }\end{array}$ \\
\hline $60^{\circ}$ & & & \\
\hline $72^{\circ}$ & & & \\
\hline $90^{\circ}$ & & & \\
\hline $120^{\circ}$ & & & \\
\hline $180^{\circ}$ & & & \\
\hline
\end{tabular}

Legenda:

$\theta$ - ângulo central;

$\frac{\theta}{2}-$ metade do ângulo central; $\quad$ sen $\frac{\theta}{2}-$ seno da metade do ângulo central;

$\mathrm{R}$ - raio da circunferência.

Pense um pouco a respeito dos valores determinados para o seno de cada valor $\theta$. Compare com o valor do seno obtido utilizando calculadora. O que você percebeu?

Continuando a nossa jornada chegamos à Europa. Aqui vários estudiosos se dedicaram à construção de tabelas de senos, dentre eles, Nicolau Copérnico. A teoria heliocêntrica do universo, proposta por Copérnico, substituiu a teoria geocêntrica defendida por Ptolomeu.

Copérnico apresentou uma tabela de semi cordas (senos) subtendendo arcos duplos. Tomou o comprimento do raio da circunferência como $\mathrm{R}=100000$ fazendo com que os valores das semi cordas fossem números inteiros. (MOREY; FARIA, 2009).

A tabela 5 apresenta parte dos valores calculados por Copérnico para $\operatorname{arcos}$ de $0^{\circ}$ a $90^{\circ}$ com intervalo de 10 em 10 minutos.

Tabela 5: Parte da tabela de semi cordas (senos) de Copérnico.

Arcos $\quad$ Semi corda




\begin{tabular}{|c|c|}
\hline (graus; minutos) & (subtendendo arcos duplos) \\
\hline $0 ; 10$ & 291 \\
\hline $0 ; 20$ & 582 \\
\hline $0 ; 30$ & 873 \\
\hline$\ldots$ & $\ldots$ \\
\hline $30 ; 0$ & 50000 \\
\hline$\ldots$ & $\ldots$ \\
\hline $45 ; 0$ & 70711 \\
\hline$\ldots$ & $\ldots$ \\
\hline$\ldots 0 ; 0$ & 86602 \\
\hline $89 ; 40$ & $\ldots$ \\
\hline $89 ; 50$ & 99998 \\
\hline $90 ; 0$ & 99999 \\
\hline
\end{tabular}

Fonte: BRUMMELEN (2009, p. 272).

Copérnico construiu a tabela de senos utilizando a geometria euclidiana assim como Ptolomeu na sua tabela de cordas. Provavelmente, o triunfo do sistema heliocêntrico sobre o geocêntrico contribuiu para que a tabela de Copérnico passasse a ser um modelo para a astronomia substituindo a de Ptolomeu.

\section{$P$ ara saber um pouco mais}

Sobre a construção de tabelas trigonométricas consulte Maor (1998), Morey (2001) e Brummelen (2009).

\section{CONSIDERAÇÕES FINAIS}

Diversos estudos no campo da Educação Matemática buscam formas diferenciadas de se trabalhar o conhecimento matemático em sala de aula. Estes estudos tratam de divulgar estratégias de ensino de Matemática através da modelagem, dos recursos tecnológicos, da resolução de problemas, da Etnomatemática, dos jogos e da História da Matemática.

Para que essas abordagens de ensino sejam viáveis, as instituições de formação de professores devem dar subsídios para que os docentes conheçam, dominem e possam utilizá-las em sala de aula com plena segurança. 
Nesse aspecto, vale destacar a disseminação dos cursos de mestrado profissional em Ensino de Ciências Naturais e Matemática como aliado no campo da pesquisa em ensino. Diferente das dissertações do mestrado acadêmico,

O trabalho de conclusão do mestrado profissional configura-se como dissertação que demonstre domínio do objeto de estudo, além da investigação aplicada à solução de problemas que possa ter impacto no sistema a que se dirige. Deve conter a descrição e discussão dos resultados, conclusões e recomendações de aplicações práticas e serem ancoradas em um referencial teórico. (FICHER, 2005, p. 28)

Além de tudo isso, o compromisso do professor com sua formação deve ser competência profissional básica, pois "A formação contínua conserva certas competências relegadas ao abandono por causa das circunstâncias." (PERRENOUD, 2000, p.155). Ou seja, as práticas pedagógicas mudam, o conhecimento evolui e os recursos cognitivos, na figura do professor, devem acompanhar essa agilidade.

Assim, ao optarmos por elaborar uma sequência de ensino de trigonometria através da abordagem histórica, além de contribuir com a disseminação desta abordagem, estamos propondo aos leitores uma reflexão acerca da forma de como a História da Matemática é abordada (caso exista) nos cursos de formação e aperfeiçoamento de professores.

O produto educacional no qual trabalhamos tem como fim apresentar o ensino de trigonometria de forma evolutiva natural. Procuramos aliar a trigonometria ao seu desenvolvimento histórico.

Para que a utilização desse produto (ou parte dele) seja viável em sala de aula, os professores interessados devem se deter a alguns requisitos básicos: ter conhecimentos em geometria, domínio de cálculos algébricos e com números irracionais, familiaridade com construções geométricas e com o estudo das funções. Sem uma preparação prévia dos participantes com relação a esses conteúdos matemáticos mencionados, nossa proposta ficará bastante limitada.

Além disso, a utilização dessa sequência de atividades pode ser aliada ao uso de alguma ferramenta educacional informatizada. No nosso curso utilizamos o software GeoGebra para a construção de algumas figuras geométricas e gráfico de função seno. Esse recurso foi utilizado como auxiliar durante a resolução e discussão de algumas tarefas das atividades. 
Caso não seja possível a utilização de algum software de geometria dinâmica, sugerimos a construção geométrica com régua e compasso para as atividades iniciais da sequência. Neste caso, o professor poderá enriquecer as informações geométricas contidas na sequência com alguns estudos complementares para desenvolvimento cognitivo dos alunos.

\section{REFERÊNCIAS}

BRITO, Arlete de J. MOREY, Bernadete B. Geometria e Trigonometria: dificuldades de professores do ensino fundamental. In: FOSSA, John A. (org): Presenças Matemáticas. Natal: EDUFRN, 2004.

BRUMMELEN, Glen van. The mathematics of the heavens and the earth: the early of trigonometry. Princeton: Princeton University Press, 2009.

FASANELLI, Florence. The political context. In: FAUVEL, John; MAANEN, Jan Van (eds.). History in mathematics education: the ICMY study. Dordrecht: Kluwer, 2002.

FERREIRA, Eduardo S. Laboratório de História da Matemática. Natal: SBHMat, 2001.

FISCHER, Tânia. Mestrado profissional como prática acadêmica. In: REVISTA BRASILEIRA DE PÓS-GRADUAÇÃO. v. 2, n. 4. 2005. p. 24-29.

KENNEDY, Edward S. História da trigonometria. Trad. Hygino H. Domingues. São Paulo: Atual, 1992.

MAOR, Eli. Trigonometric delights. New Jersey: Princeton University Press, 1998. Disponível em http://press.princeton.edu/books/maor/. Acesso em 21/09/2009.

SIU, Man-Keung. "No, I don't use history of mathematics in my class. Why?" In Proceedings HPM 2004 \& ESU4, F. Furinghetti, S.Kaijser, \& C. Tzanakis (eds.), Uppsala: Uppsala Universitet, 2004/2007. pp. 268-277.

MOREY, Bernadete B. Tópicos de história da trigonometria. Natal: SBHMat, 2001.

MOREY, Bernadete B. FARIA, Paulo Cézar de. Abordagens no cálculo do seno de $1^{o}$ : as contribuições de Ptolomeu, Al-Kashi e Copérnico. Belém: SBHMt, 2009.

PERRENOUD, Philippe. Dez novas competências para ensinar. Porto Alegre: ARTMED, 2000. 